\title{
Semantically Data Classification Analysis Algorithm for Social Media
}

\author{
Ashwini Pal \\ M. Tech Scholar \\ Department of Computer Science \& Engineering, \\ VITM, Indore, India
}

\author{
Prakash Mishra \\ Assistant Professor \\ Department of Computer Science \& Engineering, \\ VITM, Indore, India
}

\begin{abstract}
Sentiment Categorization adverts to the method approaches for classifying whether or not or no longer or not the feelings of textual content material are positive or terrible. Statistical methods supported term Presence and time period Frequency, mistreatment support Vector computing gadget are probably utilized for Sentiment Categorization. . Our technique is dependent on time interval weight programs that are used for information recuperation and sentiment categorization. It differs radically from these original methods on account that that of our mannequin of logarithmic differential time period frequency and declaration presence institution for sentiment classification. Terms with almost equal distribution in no doubt tagged documents and negatively labeled documents were categorized as a discontinue-phrase and discarded. The proportional distribution of a time interval to be categorised as stop-phrase used to be determined through an scan. We evaluated the proposed mannequin by means of evaluation it with state of art systems for sentiment classification.
\end{abstract}

\section{Keywords}

Sentiment Analysis, Opinion Mining, Support Vector Machine,Term Frequency, TF-IDF

\section{INTRODUCTION}

Data mining is an application of data processing in which expert patterns and information is extracted. This extracted information is consumed using applications and actual time programs for making choices. The net is a rich domain of knowledge, potential and leisure. Tremendous amount of consumer's entry internet, in between they are at all times still connected via their buddies making use of these offerings. Often small human grasping nature over internet invites the hidden risks. Bulling, phishing and abusing is a part of social crime that isn't recoverable making use of technological know-how. But cyber infrastructure can be prevented utilizing small efforts of approaches. The fundamental goal of this study is to overcome the social networking sites abasement and unsocial events. Accordingly a content classification scheme is required to develop for classifying these contents.

Children and adolescence international have adopted social networking web sites actively, partly considering that of the lack of kids freedoms in bodily world. Moreover technical designs of social networking websites gradual down effortless management of settings and transparency challenge the commercial use of personal expertise. Accordingly social networking filters are required to advance in which user communications are navigated and their semantically meanings are well-known. This work is indented to advance a social networking filter for textual content-headquartered contents. To enhance content centered filter some methodologies and prior equivalent works are required to analyses.

\section{LITERATURE SURVEY}

Xia Hu et al [1] examine whether or not social relation scan help sentiment analysis through proposing a Sociological strategy to handling Noisy and quick Texts (SANT) for sentiment classification. In certain, writer grants a mathematical optimization components that comprises the sentiment consistency and emotional contagion theories into the supervised learning method; and makes use of sparse learning to deal with noisy texts in Micro blogging. An empirical learn of two real-world Twitter datasets shows the superior efficiency of given framework in dealing with noisy and quick tweets.

Fei Jiang et al [2] emoticons in chinese micro weblog messages are used as annotations to routinely label noisy corpora and assemble sentiment lexicons. Facets including micro blog-certain and sentiment associated ones are introduced for sentiment classification. These sentiment indicators are priceless for chinese micro blog sentiment analysis. Opinions on a balanced dataset are performed, showing an accuracy of sixty three.9\% in a three category sentiment classification of positive, bad and neutral. The elements mined from the chinese micro blogs additionally develop the performances.

Eric Baucom et al [3] search to examine how intently Twitter mirrors the true world. Specifically, they desire to symbolize the connection between the language used on Twitter and the outcome of the 2011NBA Playoff video games. Author hypothesize that the language used by Twitter users will be valuable in classifying the users' locations combined with the current repute of which workforce is within the lead in the course of the sport. That is founded on the customary assumption that "lovers" of a crew have more confident sentiment and can for this reason use exclusive language when their workforce is doing good. They investigate this hypothesis by using labeling each and every tweet in accordance the area of the user together with the group that is within the lead at the time of the tweet.

Min Wang et al [4] propose a novel go-media Bag-of-words model (CBM) for Micro weblog sentiment evaluation. In this model, we symbolize the text and picture of a Weibo tweet as a unified Bag-of-words illustration. Headquartered on this mannequin, we use Logistic Regression to categorize the Micro blog sentiment. It performs good in the sentiment classification undertaking in view that it doesn't require the conditional dependence assumption. Additionally they use SVM and Naive Bayes to make a evaluation. Experiments on 5,000 Microblog messages exhibit that our CBM mannequin 
performs higher than textual content-situated ways. The sentiment classification accuracy on Micro weblog messages of our model is eighty\%, improved by way of four\% than the textual content-based ways.

\section{EXISTING SYSTEM}

Many unsupervised finding out approaches use present lexical resources (like Word Net) and language certain sentiment expertise (like sentiment seed words, their Synonyms and antonyms) to construct and update sentiment lexicons. [9], [10]. Very few sentiment lexicons are area precise whereas a lot of these are generalized. Cross area lexicons have been methodically increased to adapt for different associated domains if the sentiment lessons for one area are to be had [11]. Unsupervised finding out strategies assigned a generalized polarity and weight to a time period failing to seize its area special context.

Supervised learning strategies developed sentiment model knowledgeable with the help of tagged reviews. As these reviews are collection of domain-sensible tagged set, the model developed served well for special domains [12]. It used to be additionally noted in our survey that lots of the study in Sentiment evaluation has concerned about supervised finding out approaches reminiscent of Naive-Bayes, highest-Entropy and support Vector computing device (SVM) [13]. It was also marked that SVM was popularly used system for Sentiment Classification. Supervised learning strategies utterly depend upon the provision and the great of tagged dataset.

A collection of records is used as coaching set to the classifier. These records are represented as vectors. Every term in the record is an element in the vector in SVM method for text mining. Term Presence and term Frequency are two general approaches for knowledge Retrieval when representing files as vectors [8]. In term Presence procedure an aspect can take a binary value. This element is set to at least one if the term is present in document or else set to zero if the term just isn't reward in document. In time period Frequency method an aspect in the document vector is a non-terrible integer that's set to rely of the given term in a record.

TF IDF is a preferred statistical procedure to index the term as per their significance. TFIDF is centered on records and time period vectors that signify time period frequency as well as term presence [34] [35]. Time period presence would be constructed if term frequency vector is on hand however viceversa will not be possible.

$$
d^{(i)}=T F\left(w_{i}, d\right) \cdot I D F\left(w_{i}\right)
$$

Where,

$\mathrm{w}_{\mathrm{i}}=\mathrm{i}^{\text {th }}$ term. $\mathrm{d}=$ document.

$\mathrm{d}^{(\mathrm{i})}=$ TFIDF of term $\mathrm{w}_{\mathrm{i}}$ in document $\mathrm{d}$.

$\mathrm{TF}\left(\mathrm{w}_{\mathrm{i}}, \mathrm{d}\right)=$ Term Frequency of term $\mathrm{w}_{\mathrm{i}}$ in document $\mathrm{d}$. and $\operatorname{IDF}\left(\mathrm{w}_{\mathrm{i}}\right)=$ Inverse Document Frequency.

TFIDF of term wi in document $\mathrm{d}$ can be computed using "(1)". Term frequency $\operatorname{TF}\left(\mathrm{w}_{\mathrm{i}}, \mathrm{d}\right)$ is count of a term $\mathrm{w}_{\mathrm{i}}$ in document $d$. Larger value of a Term Frequency indicates its prominence in a given document. Terms present in too many documents were suppressed as these tend to be stop words. This suppression was handled by the second component IDF.

$$
I D F\left(w_{i}\right)=\log \left(\frac{|D|}{D F\left(w_{i}\right)}\right)
$$

Where,

$\operatorname{IDF}\left(\mathrm{w}_{\mathrm{i}}\right)=$ Inverse Document Frequency.

$\mathrm{w}_{\mathrm{i}}=\mathrm{i}^{\text {th }}$ term.

$|\mathrm{D}|=$ the total count of documents.

$\mathrm{DF}\left(\mathrm{w}_{\mathrm{i}}\right)=$ count of documents that contain term $\mathrm{w}_{\mathrm{i}}$.

If a term is present in all the documents then numerator equals denominator in "(2)". As a result of this $\operatorname{IDF}\left(w_{\mathrm{i}}\right)=\log 1$ which is zero. But if term occurred in relatively less number of document then $\operatorname{DF}\left(\mathrm{w}_{\mathrm{i}}\right)<|\mathrm{D}|$. As a result $\operatorname{IDF}\left(\mathrm{w}_{\mathrm{i}}\right)=\log (>1)$ which is a positive integer. Term presence vector was used for calculation of IDF. TFIDF identified important terms in given set of documents but as per Martineau and Finin top ranked index terms were not the top ranked sentimentally polarized terms [3].

\section{PROPOSED SYSTEM}

In order to resolve the identified issues in the social networking data analysis a new model is proposed in this system. The proposed data model is based on the hybrid concept of graph theory and data mining techniques. The proposed data model can be understood using the given figure 1.

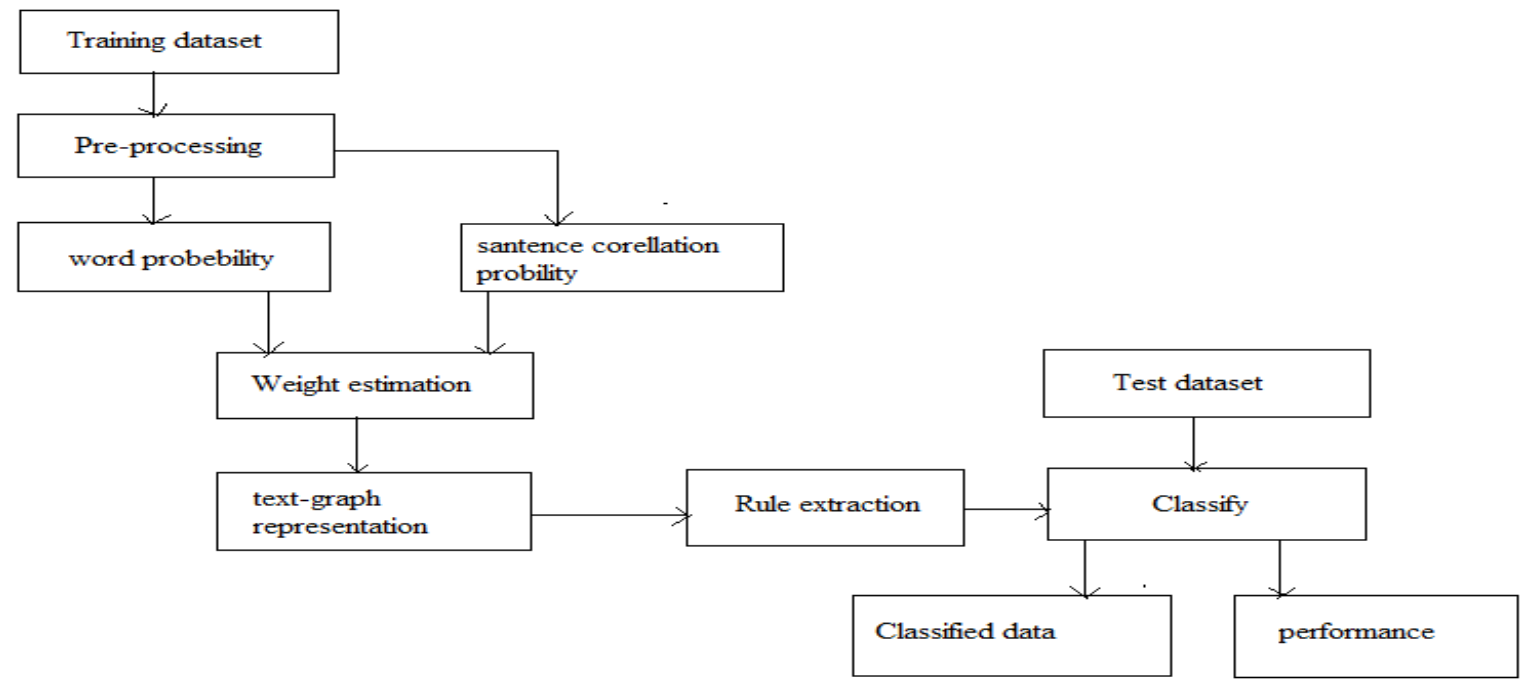

Figure 1: Proposed System 
The proposed knowledge mannequin is given in determine 1 on this diagram the model coaching and the experiment dataset classification system is simulated. Accordingly the complete knowledge model progress is carried out in two fundamental modules first training and then checking out. In coaching first the procedure receive the training information samples on which the pre-processing is carried out for the period of pre-processing the discontinue words are removed from the enter textual content and the significant text is stay from the training set. The phrase probability is majored in this measured from the text stay using the beneath given formulation.

Where the $\mathrm{N}$ is quantity of whole phrases in text files, within the identical approaches the sentence formation probability is estimated from the textual content making use of the following formulation. Where the $\mathrm{M}$ is the number of sentences in the textual content document. After that the weights for the weighted graph is computed from each the likelihood using the computed weights and the user suggestions the correlation graph is developed in additional steps and making use of these weighted graphs the classification principles are developed. These ideas units are consumed to identify the sentiments of the phrases worried within the micro-weblog textual content.

In connection with the occurrences of infrequent words, oneof-a-kind versions of TFIDF ratings of phrases, indicating the change in occurrences of phrases in extraordinary classes (confident or bad experiences), have been urged by Paltoglou and Thelwall [14]. They surveyed many time period weighting procedures as well proposed "smart" and "BM25" time period weighting strategies for sentiment classification. TFIDF identified important phrases in given set of documents but as per Martineau and Finin top ranked index phrases were not the top ranked sentimentally polarized phrases [3]. Martineau and Finin constructed vectors to classify a time period headquartered on term frequency vector as well as term presence vectors. Not like TFIDF which used single time period presence vector, two vectors were separately constructed for presence in positively tagged files and negatively tagged files [3].

$$
V_{t d}=C_{t d} \times \log \left(\frac{\left|N_{t}\right|}{\left|P_{t}\right|}\right)
$$

Where,

$V_{t d}=$ Polarity of term $t$ in document $d$.

$\mathrm{C}_{\mathrm{td}}=$ count of a term in a given document.

$\left|\mathrm{N}_{\mathrm{t}}\right|=$ count of negatively tagged documents with term $\mathrm{t}$.

$\left|P_{t}\right|=$ count of positively tagged documents with term $t$.

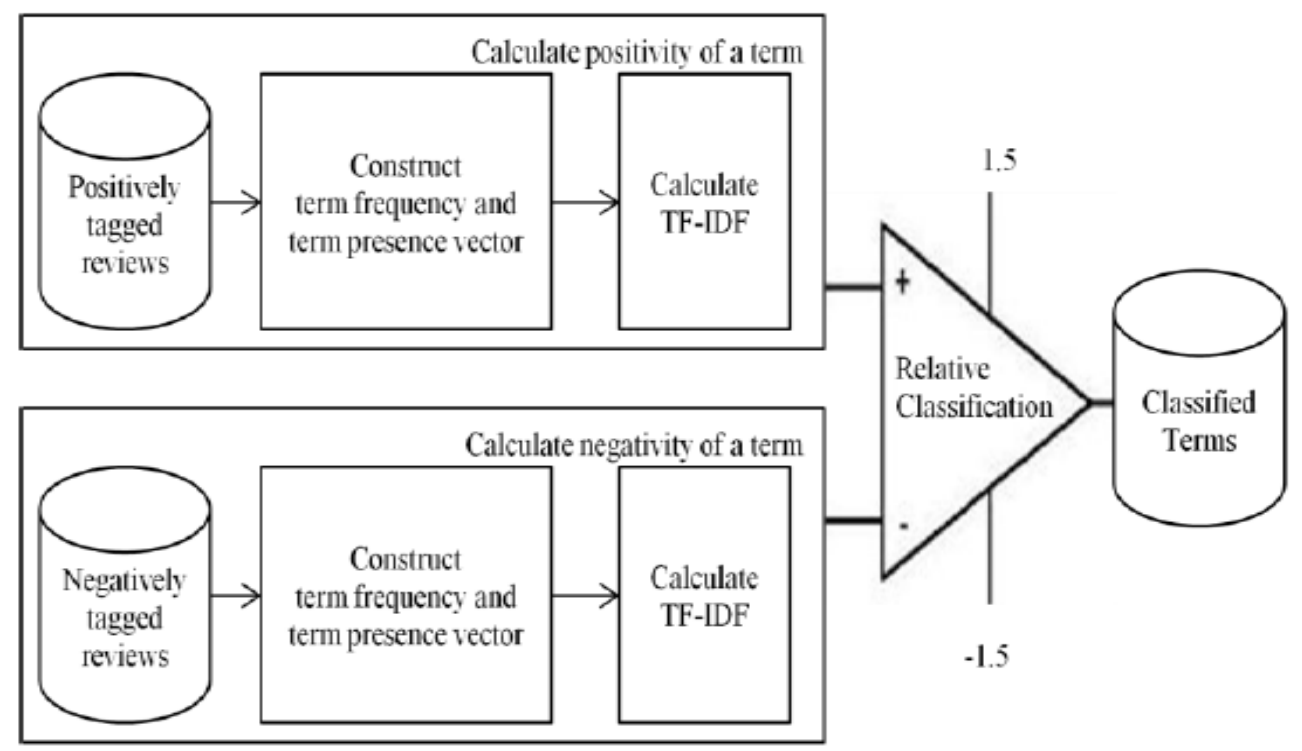

Fig. 2. The proposed

Extra value was once given in "(3)" to terms that took place more often than not. The later part of the mannequin period. Term presence rely of a time period used to be number of files that time period was once gift) component back a terrible price if a term came about in more number of positively tagged documents as compared to negatively tagged files and vice-versa. If a term was present in equal quantity of optimistic and terrible file then this element returned zero. Considering the fact that this value used to be elevated with $\mathrm{Ctd}$, ensuing Vtd value used to be also grounded. These terms were classified as discontinue words. Delta TFIDF lower back a poor value if the time period was once categorized as optimistic and vice-versa. It considered total depend of phrases in all records ignoring the frequency distribution of terms across positively and negatively tagged files. For instance if a term was reward in additional number of negatively tagged records as compared to positively tagged file, term was labeled as negative. Despite the fact that the time period was once present in less number of positively tagged documents, its frequency count in these positively tagged records could also be more which contributed to Ctd section. This incorrectly boosted the Vtd price. Ctd being frequency count of phrases over all of the records didn't accurately relates to 2 nd a part of the model that dealt with distribution of presence. To calculate polarity of ith time period summation of ith element of the vectors used to be taken where) used to be usual. Sum of Ctd which was continuously a positive number acted as a boosting element.

Our mannequin SentiTFIDF works on the principle logarithmic proportion of TFIDF of a time period throughout positively tagged files and negatively tagged files. If the TFIDF of a time period in positively tagged records is larger 
than TFIDF of equal time period in negatively tagged documents the time period is assigned confident polarity and vice-versa.

\section{SentiTFIDF based on relative TFIDF}

Figure 2 presents the method glide of SentiTFIDF. It may be divided into three parts. In first part the positivity of a term is calculated. In a similar fashion negativity of a term is calculated in second section. Third part classifies the time period as positive, bad or impartial centered on its share of positivity and negativity calculated in earlier steps.

\section{RESULT}

A record of terms that occurred in the records was prepared. A term is entered only once on this time period record despite the fact that it's going to appear may just instances in documents. A report vector was once developed for every report. Each ith aspect on this vector was rely of ith term in this record. If a term in time period record was now not gift within the file the rely associated with that term used to be set to zero. These vectors were used to calculate time period polarity for the terms in the term record. A term was categorized either as positive or bad or neutral. Our mannequin as confident if complete number of constructive terms within the document were more than poor terms categorized a report. Similarly a file used to be categorized as terrible if complete quantity of negative terms within the report have been more than constructive phrases.

\section{CONCLUSION}

Sentiment detection has a broad range of applications in expertise systems, including classifying studies, summarizing overview and different real time applications. There are feasible to be a number of extraordinary purposes that's not acknowledged. It's determined that sentiment classifiers are severely keen about domains or topics. From the above work it's evident that neither classification mannequin systematically outperforms the reverse, exclusive types of options have designated distributions. It's also found that differing types of choices and classification algorithms square measure mixed in a cheap method so that you could beat their individual drawbacks and have the benefit of each other"s deserves, and finally increase the sentiment classification performance.

\section{REFERENCES}

[1] Hanjun Lee Business School, Korea "The Influence Of Negative Emotions In An Online Brand Community On Customer Innovation Activities " 2014 47th Hawaii International Conference on System Science -978-14799-2504-9/14 \$31.00 @ 2014 IEEE

[2] Xia Hu, Lei Tang, Jiliang Tang, Huan Liu, "Exploiting Social Relations for Sentiment Analysisin Microblogging", permission and/or a fee.WSDM '13, February 4-8, 2013, Rome, Italy.Copyright 2013 ACM 978-1-4503-1869-3/13/02

[3] Fei Jiang, Anqi Cui, Yiqun Liu, Min Zhang, and Shaoping Ma, "Every Term Has Sentiment:Learning from Emoticon Evidencesfor Chinese Microblog Sentiment Analysis",c Springer-Verlag Berlin Heidelberg 2013

[4] Eric Baucom,AzadeSanjari, Xiaozhong Liu,Miao Chen, "Mirroring the Real World in Social Media: Twitter,Geolocation, and Sentiment Analysis",Copyright 2013 ACM 978-1-4503-2415-1/13/10
[5] Min Wang,Donglin Cao, Lingxiao Li,Shaozi Li, RongrongJi, "Microblog Sentiment Analysis Based on Cross-mediaBag-of-words Model",ICIMCS'14, July 10 12, 2014, Xiamen, Fujian, China.Copyright 2014 ACM 978-1-4503-2810-4/14/07

[6] Felipe Bravo-Marquez, Marcelo Mendoza,Barbara Poblete, "Combining Strengths, Emotions and Polarities forBoosting Twitter Sentiment Analysis",WISDOM'13, August 11 2013, Chicago, IL, USACopyright 2013 ACM 978-1-4503-2332-1/13/08

[7] Pedro Calais Guerra, Wagner Meira Jr.,Claire Cardie, "Sentiment Analysis on Evolving Social Streams:How Self-Report Imbalances Can Help",WSDM'14, February 24-28, 2014, New York, New York, USA.Copyright 2014 ACM 978-1-4503-2351-2/14/02

[8] E. Cambria, B. Schuller, Y. Xia, and C. Havasi, "New Avenues in Opinion Mining and Sentiment Analysis," Intelligent Systems, IEEE, vol.28, no.2, pp. 15-21, 2013.

[9] S. Baccianella, A. Esuli, and F. Sebastiani, "Senti WordNet 3.0: An Enhanced Lexical Resource for Sentiment Analysis and Opinion Mining," In Proc. of 7th Int'1 Conf. on Language Resources and Evaluation, pp 2200-2204, 2010.

[10] A. Neviarouskaya, H. Prendinger, and M. Ishizuka, "SentiFul: A Lexicon for Sentiment Analysis," In IEEE Trans. On Affective Computing, vol. 02, issue no. 01, pp. 22-36, 2011.

[11] Bollegala, D. Weir, and J. Carroll, "Crossm Domain Sentiment Classification using a Sentiment Sensitive Thesaurus,"Knowledge and Data Engineering, IEEE Transactions, vol.25, issue no.08, pp. 1, 0

[12] R. Xia and C. Zong, "A POS-based Ensemble Model for Cross-domain Sentiment Classification," In Proc. of 5th Int'l Joint Conf. on Natural Language Processing, pp. 614-622, 2011.

[13] K. Ghag and K. Shah, "Comparative analysis of the techniques for Sentiment Analysis," In Proc. of Int'l Conf. on Advances in Technology and Engineering, pp. 1-7, 2013.

[14] G. Paltoglou and M. Thelwall, "A study of Information Retrieval weighting schemes for sentiment analysis," In Proc. of 48th Annual Meeting of the Association for Computational Linguistics, pp. 1386-1395, 2010.

[15] B. Pang, L. Lee, and S. Vaithyanathan, "Thumbs up? sentiment classification using machine learning techniques," In Proc. of Conf. on Empirical Methods in Natural Language Processing, pp 79-86, 2002.

[16] T. Mullen and N. Collier, "Sentiment analysis using support vector machines with diverse information sources,' In Proc. of Conf. on Empirical Methods in Natural Language Processing," pp 412-418, 2004.

[17] Charles E. Osgood, George J. Suci, and Percy H. Tannenbaum. The measurement of meaning, 2nd ed. University of Illinois Press Urbana, 1967.

[18] P. D. Turney, "Thumbs up or thumbs down? semantic orientation applied to unsupervised classification of reviews," In Proc. of the 40th Annual Meeting on Association for Computational Linguistics ACL, pp 417 424, 2002. 
[19] O.F. Zaidan, J. Eisner, and C.D. Piatko, "Using Annotator Rationales to Improve Machine Learning for Text Categorization," In Proc. of Conf. of North American Chapter of the Association for Computational Linguistics, pp 260-267, 2007.

[20] Rudy Prabowo and Mike Thelwall,"Sentiment analysis: A combined approach," Journal of Informetrics,3(2):143-157, 2009.
[21] Wei-Hao Lin, Theresa Wilson, Janyce Wiebe, and Alexander Hauptmann, "Which side are you on? identifying perspectives at the document and sentence levels," In Proceedings of the Conferenceon Natural Language Learning, 2006 .

[22] Hugo Liu., "MontyLingua: An end-to-end natural language processor with common sense". Technical report,MIT, 2004. 